\title{
Image Denoising by Averaging of Piecewise Constant Simulations of Image Partitions
}

\author{
Max Mignotte
}

\begin{abstract}
This paper investigates the problem of image denoising when the image is corrupted by additive white Gaussian noise. We herein propose a spatial adaptive denoising method which is based on an averaging process performed on a set of Markov Chain Monte-Carlo simulations of region partition maps constrained to be spatially piecewise uniform (i.e., constant in the grey level value sense) for each estimated constant-value regions. For the estimation of these region partition maps, we have adopted the unsupervised Markovian framework in which parameters are automatically estimated in the least square sense. This sequential averaging allows to obtain, under our image model, an approximation of the image to be recovered in the minimal mean square sense error. The experiments reported in this paper demonstrate that the discussed method performs competitively and sometimes better than the best existing state-of-the-art wavelet-based denoising methods in benchmark tests.
\end{abstract}

Index Terms-Image denoising, Markov chain Monte-Carlo (MCMC) simulations, Markovian segmentation.

\section{INTRODUCTION}

D UE to the imperfection of image acquisition systems and transmission channels, images are often corrupted by noise. This degradation leads to a significant reduction of image quality and then makes more difficult to perform high-level vision tasks such as recognition, 3-D reconstruction, or scene interpretation. In most cases, this corruption is commonly modeled by a zero-mean additive white Gaussian random noise leading to the following additive degradation model

$$
y=x+n
$$

where $y, x$, and $n$ represent respectively the noisy observed image (of size $N$ pixels), the clean image and the corrupting additive white stationary Gaussian noise (AWGN) with variance $\sigma^{2}$. In this context, the problem of recovering $x$ from $y$ is usually known as a denoising problem.

To this end, there has been considerable interest, over the last decade, in wavelet-based denoising methods [1]-[21]. In these schemes, the basic idea consists in projecting the noisy image onto a properly selected orthogonal set of basis functions and,

Manuscript received September 20, 2005; revised July 26, 2006. The associate editor coordinating the review of this manuscript and approving it for publication was Dr. Thomas S. Denney, Jr.

The author is with the Département d'Informatique et de Recherche Opérationnelle (DIRO), Université de Montréal, Montréal, QC H3C 3J7 Canada (e-mail: mignotte@iro.umontreal.ca).

Color versions of one or more of the figures in this paper are available online at http://ieeexplore.ieee.org.

Digital Object Identifier 10.1109/TIP.2006.887729 before reconstruction, in finding some kind of threshold that will tend to remove more noise (mostly represented by low wavelet coefficients) than important image edge information (mostly represented by high-value coefficients).

Many researchers have then investigated wavelet-based denoising tools with variation on the basic procedure (and with some notable improvements), including shrinking instead of thresholding, Bayesian thresholds, translation invariant transforms, modification or level-dependent or adaptive choice of thresholding functions, overcomplete decompositions (e.g., curvelet transform [7]), etc., to name a few.

Among the research works that do not use wavelet based transforms or, more generally, the reconstruction principle using projections onto set of basis functions, we can cite the numerous existing local smoothing filters such as the Gaussian spatial filter and its variants such as the adaptive smoothing filter proposed by Lee [22]. More recently, we can cite the fractal-based denoising procedure proposed in [23] for which one can reasonably think that most of the denoising process is due to the decimation associated with the contractive spatial maps, used in the fractal transform. A wavelet extension of this work, with some improvements, has also been recently proposed in [24]. We can also cite the original non local means denoising algorithm recently proposed in [25] which chooses for each pixel of the denoised estimated image a weighted average of grey level values presenting a close neighborhood configuration. In this procedure, the denoising process (especially well suited for denoising images containing textural patterns) is due to the regularity assumption that self similarities of neighborhoods exist in a real image and that a neighborhood configuration can then be efficiently used for predicting the central value of the pixel, as was first shown in [26] for texture synthesis with a somewhat similar non parametric sampling strategy.

To the best of our knowledge, little attention has been given to the use of segmentation(s) for the purpose of image denoising. Nevertheless, image denoising and segmentation remains two related, fundamental problems. Some researchers even think that these two problems are dual and complementary and should be treated in parallel as it was put in a mathematical form in the seminal paper proposed by Mumford and Shah [27]. The segmentation process allows to capture the underlying geometrical structure hidden in real images corrupted by random noise and this information may efficiently be exploited by the denoising strategy.

Among the rare segmentation-based denoising approaches, we can cite the ones using, once again, wavelet transforms. In these models, the segmentation is generally exploited to make the wavelet-based denoising procedure spatially adaptive to the 
image behavior. It is indirectly the case of the numerous statistical denoising methods using a particular (sometimes local) prior distribution mixture model in order to segment the wavelet coefficients and to exploit their local statistics related to the local behavior of the image. In this context, a certain choice of prior mixture model for the wavelet coefficients implies a statistical estimate that produces the (possibly multiple and level dependent) spatially adaptive thresholding or shrinkage functions [5], [15]-[21]. It is also the case of the procedure described in [14] which proposes to denoise each preliminary segmented region independently of the others with a multiple compaction domain wavelet-based algorithm. It is also the case of the method described in [28] which applies two different wavelet coefficient distribution models for each of the two segmented classes of region generally present in any given image; namely, edges/texture regions and flat areas. Among the denoising approaches combining spatial filtering and segmentation, we can cite the interesting Filtering Using Explicit Local Segmentation (FUELS) algorithm proposed by Seeman and Tisher in [29]. In this approach, each overlapping squared neighborhood encompassing the current pixel to be denoised is filtered using only the intensity level of the pixels belonging to the same class (segment) of the considered central pixel. To this end, a binary segmentation, involving a (minimum mean square error) thresholding procedure is precomputed on each neighborhood region before the spatial filtering process. We can also cite [30] in which a watershed analysis (i.e., a binary segmentation process that separates the pixels into high-gradient and lower gradient connected group of pixels) is exploited by the spatial filtering process. Let us note that segmentation and denoising can also be carried out in parallel in order to automatically estimate an adaptive threshold which enables the correct segmentation of nonuniformly illuminated scenes as proposed in [31] or sequentially in order to constrain other restoration problems such as the deconvolution problem [32], [33].

In this paper, we propose to use an adaptive denoising method which relies on simulations of oversegmentation maps. More precisely, these simulations of oversegmentations allow to perform a double averaging process. The first one is a spatial local averaging process and is performed on estimated constant-value regions of the input image. This averaging allows to reduce the Gaussian noise while preserving contours of the image. This first averaging process is in accordance with our image model which expresses the fact that any image can be well approximated as a union of a number of nonoverlapping and distinct regions of uniform (i.e., constant) grey level value. In our application, this region partition map is obtained from an unsupervised Markovian oversegmentation using a classical local Potts prior and appropriate Gaussian conditional luminance distributions, automatically estimated in the least-square (LS) sense. The second one is a sequential averaging process and is performed on a Markov chain sequence of simulations of these above-mentioned segmentation maps according to its posterior distribution model. This sequential averaging allows to obtain, under our image model, an approximation of the image to be recovered in the minimal mean-square sense error (MSE). The performance of this denoising scheme will be demonstrated and some comparisons with the best available wavelet-based images denoising methods will be given in order to illustrate the effectiveness of the proposed model.

The remainder of this paper is organized as follows. The proposed denoising model is described in Section II. In Section III, we present the procedure of region partition. Section IV details the empirical estimators of the regularization parameters of our procedure. Finally, Section V presents a set of experimental results and comparisons with existing denoising techniques.

\section{PROPOSED APPROACH}

Our goal, in this denoising problem, is to obtain an estimate $\hat{x}$ of $x$ from $y$ such that the expectation of the MSE, i.e., $E\left[\|x-\hat{x}\|^{2} / N\right]$ is minimized. In fact, this MSE measure, which is the simple Euclidean distance between the original and denoised estimated image, is also commonly proposed in the denoising community in order to quantitatively measure the achieved performance improvement of a denoising technique leading to the well-known peak signal-to-noise ratio (PSNR) expressed in decibels thanks to the relation $20 \log _{10}[255 / \sqrt{\mathrm{MSE}}]$. Under some assumptions, the optimal regularization scheme, in the minimum MSE sense, is provided by the linear Weiner filter [34]. However, this optimality is obtained under the assumption that the image and noise processes are second-order stationary (in the random process sense). This assumption is not valid for most real-world images since statistics may change across an image due to the presence of spatially localized phenomena such as edges, textures, homogeneous regions, etc. For such spatially nonstationary images, the Weiner filtering approach is inappropriate.

In our approach and in order to regularize the solution (i.e., the denoised estimated image), we restrict the admissible solutions, $x$, to piecewise constant (smooth) images. More precisely, we define a regularized solution as a sum of $N_{\mathrm{R}} 2-\mathrm{D}$ piecewise constant functions (i.e., regions) as proposed in [22]. In this model a version of $x=\left(x_{1}, \ldots, x_{N}\right)^{T}$ can be represented by

$$
x_{i}=\sum_{j=1}^{N_{\mathrm{R}}} \mu_{j} I_{\left\{i \in \mathcal{R}_{j}\right\}} \quad \text { for } \quad i=1, \ldots, N
$$

where $\mu_{j}$ denotes the mean grey value of the $j$ th region, $N$ is the total number of pixels, $i \in \mathcal{R}_{j}$ means "the $i$ th pixel is in the $j$ th region," $I_{E}$ is the indicator function for the event $\mathrm{E}$ and $N_{\mathrm{R}}$ is the total number of regions. In our formulation, we have chosen to use single indexing (rather than double indexing) for labelling pixel coordinates. This image model simply expresses the fact that any (real-world or synthetic) images can be approximated by an union of a number of nonoverlapping and distinct regions of uniform (i.e., constant) grey level value. This assertion is true, without error, for region of size 1 pixel and a good approximation for regions with at most $W_{r}=10-20$ pixels (see Section V and Fig. 1 for confirmation).

Under this (regularized) image model, the proposed denoising approach then consists in defining the estimated image $\hat{x}$, in the minimal MSE sense, by

$$
\hat{x}=E[x \mid y]
$$




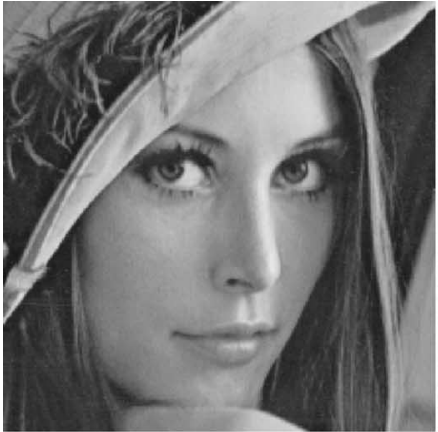

(a)

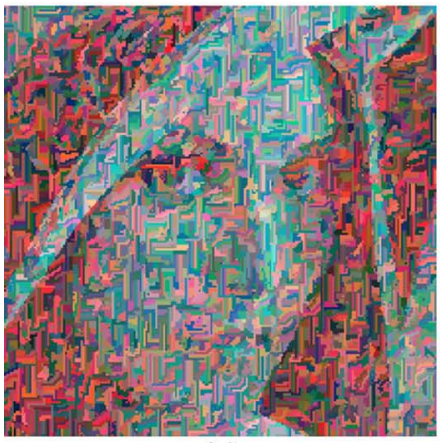

(e)

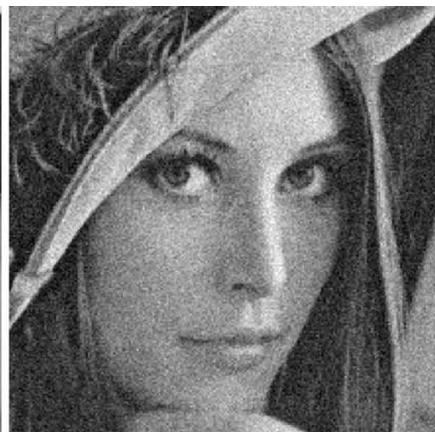

(b)

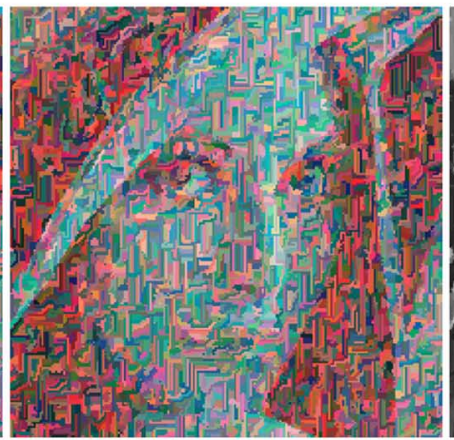

(f)

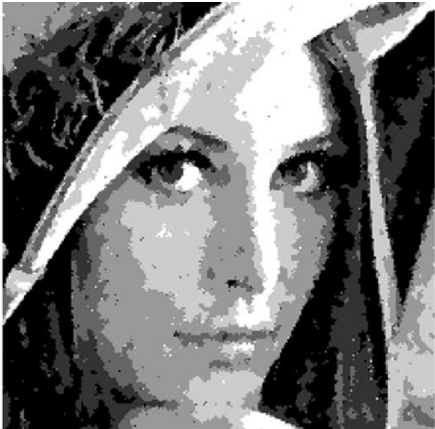

(c)

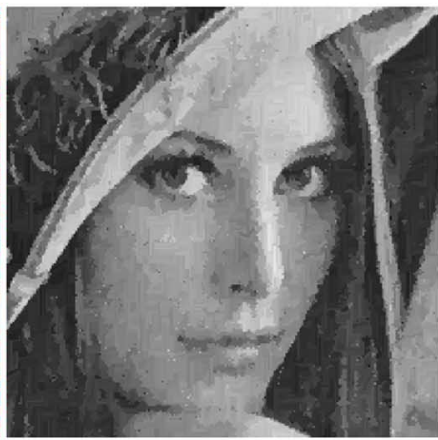

(g)

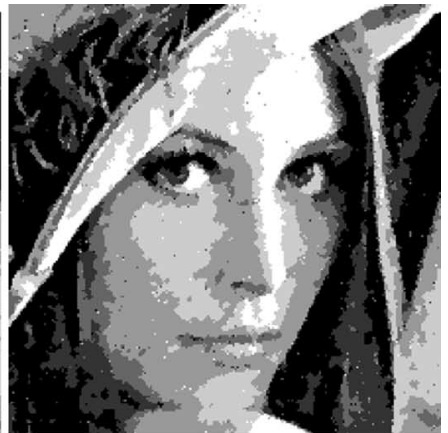

(d)

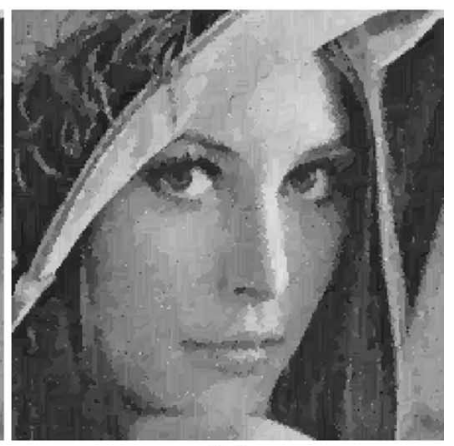

(h)

Fig. 1. (a) Cropped image from the original $512 \times 512$ Lena image. (b) Noisy image with AWG noise with variance $\sigma^{2}=15$ (MSE $=224.6$, PSNR $=$ $24.62 \mathrm{~dB}$ ). (c) Unsupervised nine-class segmentation. (d) Another unsupervised Markovian nine-class segmentation (obtained for another seed). (e) Partition into regions of the segmentation presented in (c) with $W_{r}=15$. (f) Partition into regions of the segmentation map presented in (d) with $W_{r}=15$ showing 26142 regions with variable sizes ( 1 to 15 pixels for the original non cropped Lena image) and an average size of 10 pixels size. (g) Realization $x_{(1)}$ based on the partition into regions presented in $(\mathrm{e})(\mathrm{MSE} \approx 86)$. (h) Realization $x_{(2)}$ based on the partition into regions presented in $(\mathrm{f})(\mathrm{MSE} \approx 87)$.

in which the expectation is only over the admissible solutions. Thanks to the law of large numbers, (3) is approximated by

$$
\hat{x}=\frac{1}{N_{\mathrm{S}}}\left(x_{(1)}+\cdots+x_{\left(N_{s}\right)}\right)
$$

where $x_{(i)}, \quad i=1, \ldots, N_{\mathrm{S}}$ are admissible realizations of $X$ (with the convention that $X$ is the random process associated to the particular realization $x$ ) according to a distribution which is not analytically defined but which gives a realization $x$ of $X$, under the image model given in (2), by the following procedure.

A simple way of simulating a regularized realization $x$ given the observed and noisy data $y$ consists in simulating a partition into regions $\mathcal{R}$ of this image and then in replacing each pixel value belonging to each region $\mathcal{R}_{j}$ of $\mathcal{R}$ by its grey level mean according to (2). To this end, $\mathcal{R}$ is obtained from an oversegmentation of $y$ by breaking its connected regions (i.e., the set of connected pixels belonging to a same class) into small disjoint pieces of no more than $W_{r}$ pixels. Substituting (2) in (4), we obtain

$$
\begin{aligned}
\hat{x}_{i} & =\frac{1}{N_{\mathrm{S}}}\left(\sum_{j=1}^{N_{\mathrm{R}(1)}} \mu_{j(1)} I_{\left\{i \in \mathcal{R}_{j(1)}\right\}}+\right. \\
& \left.\cdots+\sum_{j=1}^{N_{\mathrm{R}\left(N_{s}\right)}} \mu_{j\left(N_{s}\right)} I_{\left\{i \in \mathcal{R}_{j\left(N_{s}\right)}\right\}}\right) \text { for } i=1, \ldots, N
\end{aligned}
$$

where $N_{\mathrm{R}(i)}$ is the total number of regions of the ith simulations. $\mathcal{R}_{j(i)}$ and $\mu_{j(i)}$ denote, respectively, the $j$ th region from partition $i$ and the mean grey value of the $j$ th region from partition $i$.

We explain in the following section how to obtain this oversegmentation according to an MRF model, how to subdivide this segmentation into small disjoint pieces and finally how to estimate the parameter vector $\boldsymbol{\mu}=\left\{\mu_{j(k)}\right\}, k=1, \ldots, N_{s}$, $j=1, \ldots, N_{\mathrm{R}(k)}$. Note that the same approach could be used with various segmentation methods.

\section{UNSUPERVISED REGION PARTITION PROCEDURE}

\section{A. Unsupervised Markovian Oversegmentation}

To this end, we have considered the monoscale version of the Markovian estimation model of segmentation into $K$ classes described in [35], and already successfully applied to noisy sonar images. In this denoising application we take a Gaussian law as degradation model to describe the luminance distribution within each class. In this Bayesian approach, the MRF-based estimation problem of segmentation consists first in inferring the parameters of the grey value distribution on each class $\hat{\Phi}_{y}$ which is achieved thanks to an iterative method called iterative conditional estimation (ICE) [36] which gives the best estimation of $\hat{\Phi}_{y}$ in the LS sense. This ICE procedure is initialized by a $\mathrm{K}$-means clustering procedure [37] as proposed in [35] and a Gibbs sampling is used for the simulation process. This iterative procedure allows to both estimate $\hat{\Phi}_{y}$ and to obtain, at con- 


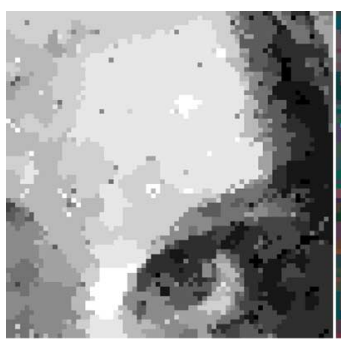

(a)

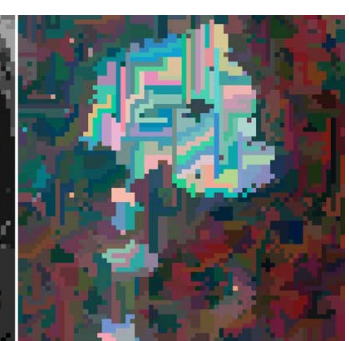

(b)
Fig. 2. (a) Segmentation into 12 classes of a noisy version of Lena image ( $\sigma=20$ ) obtained at convergence of the ICE procedure. (b) Partition into regions of the segmentation map presented in (a) exhibiting different randomly chosen subdivision patterns, of $W_{r}=15$ pixels maximal size, for the different regions associated to one (highlighted) class of the segmentation. The patterns of subdivision are randomly chosen as being vertical horizontal or diagonal atomic structures.

vergence, ${ }^{1}$ a segmentation map based on $P_{Z \mid Y, \hat{\Phi}_{y}}(z \mid y)$. In our application, we take $\beta_{i}(i=1, \ldots, 4)$, the parameters of the a priori model and the number of classes $K$ as a function of the variance of the AWG noise (this will be made explicit in Section V). Fig. 1(c) and (d) displays two examples of unsupervised nine-class segmentation of $y$, exploiting parameters $\hat{\Phi}_{y}$ estimated with the stochastic ICE procedure (for a different seed for each example).

\section{B. Partition Into Regions}

We now exploit this oversegmentation ${ }^{2}$ in order to get a reliable partition $\hat{\mathcal{R}}$ of the image into homogeneous regions. To this end, we simply search the set of disjoint regions (i.e., the set of connected pixels belonging to the same class). In order to limit regions with a large number of pixels, which could be in accordance with our image model, we subdivide all the regions with more than $W_{r}$ pixel size. In our application, we take $W_{r}=\sigma$ which induces that the segmentation-based spatial averaging process will be proportional to the standard deviation of the Gaussian degradation model. In order to avoid visual artifacts, we subdivide each region of each segmentation with a different subdivision pattern (see Fig. 2 and Algorithm 2). The parameter vector $\boldsymbol{\mu}=\left\{\mu_{j(k)}\right\}$, is then simply estimated by

$$
\mu_{j(k)}=\frac{1}{\left|\mathcal{R}_{j(k)}\right|} \sum_{i=1}^{N} y_{i} I_{\left\{i \in \mathcal{R}_{j(k)}\right\}}
$$

where $\left|\mathcal{R}_{j(k)}\right|$ denotes the cardinality of the $j$ th region from partition $k$. Due to the stochastic aspect of the simulation process we obtain different possible oversegmentations [cf. Fig. 1(c) and (d)] and, thus, different partitions into regions of the same input image [see Fig. 1(e) and (f)]. These simulations of region maps are differently partitioned. Nevertheless, the localized characteristics of the image such as the main structures, the edges and the details are preserved by these partitions which define a set of possible low-level representations of the input image. These

\footnotetext{
${ }^{1}$ To this end, we can use an adaptive criterion for the number of iterations as the one proposed in [35] which is based on an indicator of the "stability" of the procedure.

${ }^{2}$ An oversegmentation is a partition of the image in which small regions are favored and which is beyond what is needed to divide the image semantically into different objects of interest. The different elements or atomic regions of an oversegmentation are sometimes called superpixels or segments.
}

(a)

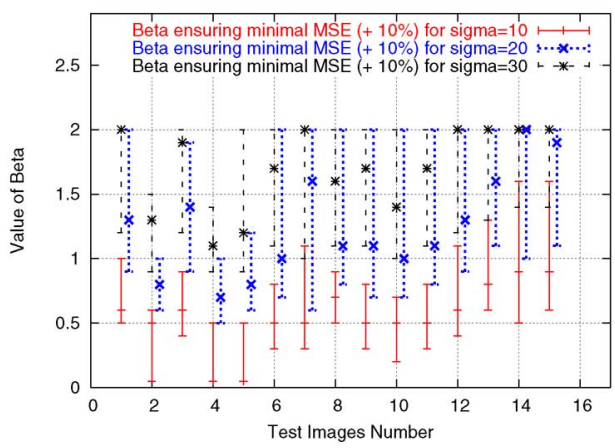

(b)

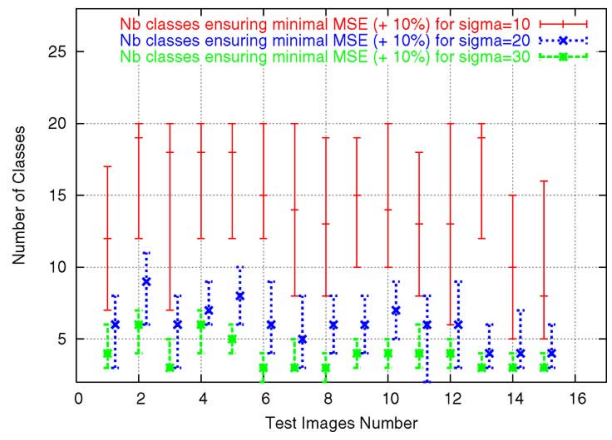

Fig. 3. Optimal value ensuring the minimal MSE denoising measure (and interval for ensuring MSE ; minimal MSE $+10 \%$ ) for a set of 15 test images and for (a) $\beta$ the regularization parameter of the posterior distribution (with $N_{\mathrm{S}}=20$ and $K=6$ ) and (b) the value of $K$ (with $\beta=1.0$ and $N_{\mathrm{S}}=20$ ).

sets of competing partitions allows to define realizations of $x$ according to our image model given in (2) [see Fig. 1(g) and (h)] which are already a denoised version, due to the spatial averaging process on each region. This can be noticed simply by visual evaluation or by comparing the MSE result of the noisy image (MSE $=225$ ) with the one obtained after the spatial averaging process $\left(\mathrm{MSE} \approx 86\right.$ ) for each of $x_{i}$. This spatial local averaging process, performed on estimated regions of the input image reduces the noise while preserving contours of the image but also introduces some visual artifacts due to the inherent false contours generated by the segmentation process. These artifacts (and, consequently, the MSE result) will be all the more reduced than the sequential averaging process leading to the final denoised estimate, will be done on a great number of simulations.

\section{ESTIMATION OF REGULARIZATION PARAMETERS}

First, we have tested the sensitivity of the two following parameters of our segmentation-based denoising model which both act as a regularization parameter, namely:

1) $\beta\left(=\beta_{1}=\ldots=\beta_{4}\right)$, the regularization or prior term of our prior distribution and

2) $K$, the number of classes of the Markovian segmentation model

on the result of the minimal MSE (or equivalently the maximal PSNR) measure for the denoising problem. Fig. 3 shows the optimal value for the parameter $\beta$ and $K$ the number of classes ensuring the minimal MSE for a set of $15(512 \times 512$ black and white) test images (training population). First, we can note that the optimal value for $\beta$ has to be around $\beta=0.3-0.8$ for a degradation model with variance $\sigma=10$, around $\beta=1.0$ for 


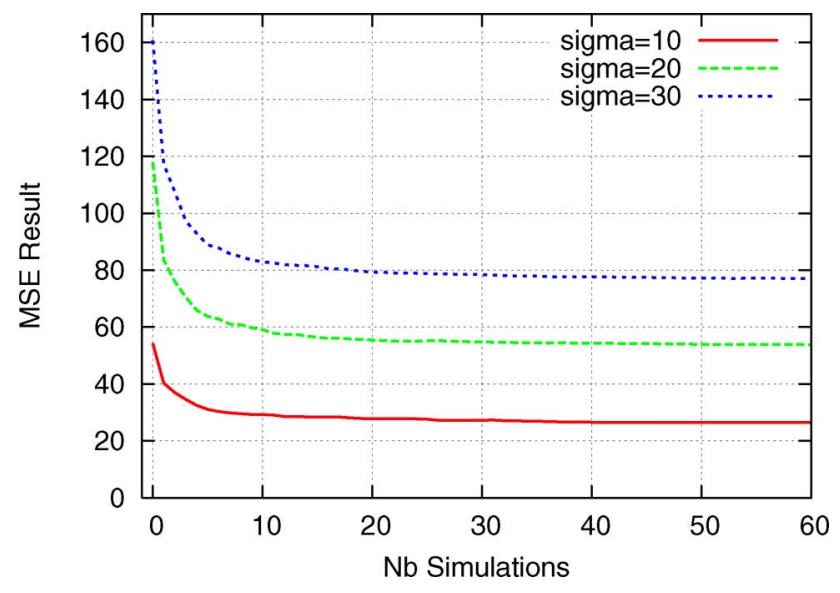

Fig. 4. Evolution of the MSE measure along the number of simulations for the denoising of the Lena image (and for $\sigma=\{10,20,30\}$ ).

$\sigma=20$ and around $\beta=1.4$ for $\sigma=30$ which leads to the following empirical estimation model for $\beta$

$$
\hat{\beta}=\max \left\{0.25, \frac{\sigma}{20}\right\} \text {. }
$$

Second, we can also note that the optimal value for $K$ is around $K=10-15$ for a degradation model with variance $\sigma=10$, around $K=6-8$ for $\sigma=20$ and around $K=4-6$ for $\sigma=30$ which leads to the following empirical estimation model for $K$

$$
\hat{K}=\max \left\{6,\left[\frac{\text { Number of grey levels }=256}{2 \sigma}\right]\right\}
$$

where $[x]$ denotes the superior integer part of $x$ and a minimal number of classes (six in or application) is required in order to avoid visual artifacts created by false contours generated by a segmentation with a low number of classes, i.e., segmentations computed on images corrupted by important noise. In order to minimize visual artifacts and the MSE result, we have empirically noticed that a nonfixed number of classes, and more precisely a random value of the number of classes between 3 , and the maximal number given in (8) allowed us to improve the denoising result. We, thus, use a Gibbs sampling with $K$, randomly chosen in

$$
K \in\left[3, \max \left\{6, \frac{256}{2 \sigma}\right\}\right]
$$

As already said in Section III-B, we take $\hat{W}_{r}=\sigma$ which induces that the segmentation-based spatial averaging process will be proportional to the standard deviation of the Gaussian degradation model (this value is empirically determined and ensures minimal MSE denoising results on our training image database). The required number of simulation is also empirically set. To this end Fig. 4 shows the evolution of the MSE measure along the number of simulations for the denoising of the Lena image for, respectively, the three following degradations models: $\sigma=\{10,20,30\}$. This graph show us that a convergence is achieved in less than 60 iterations. This number of simulations will be used in our tests.
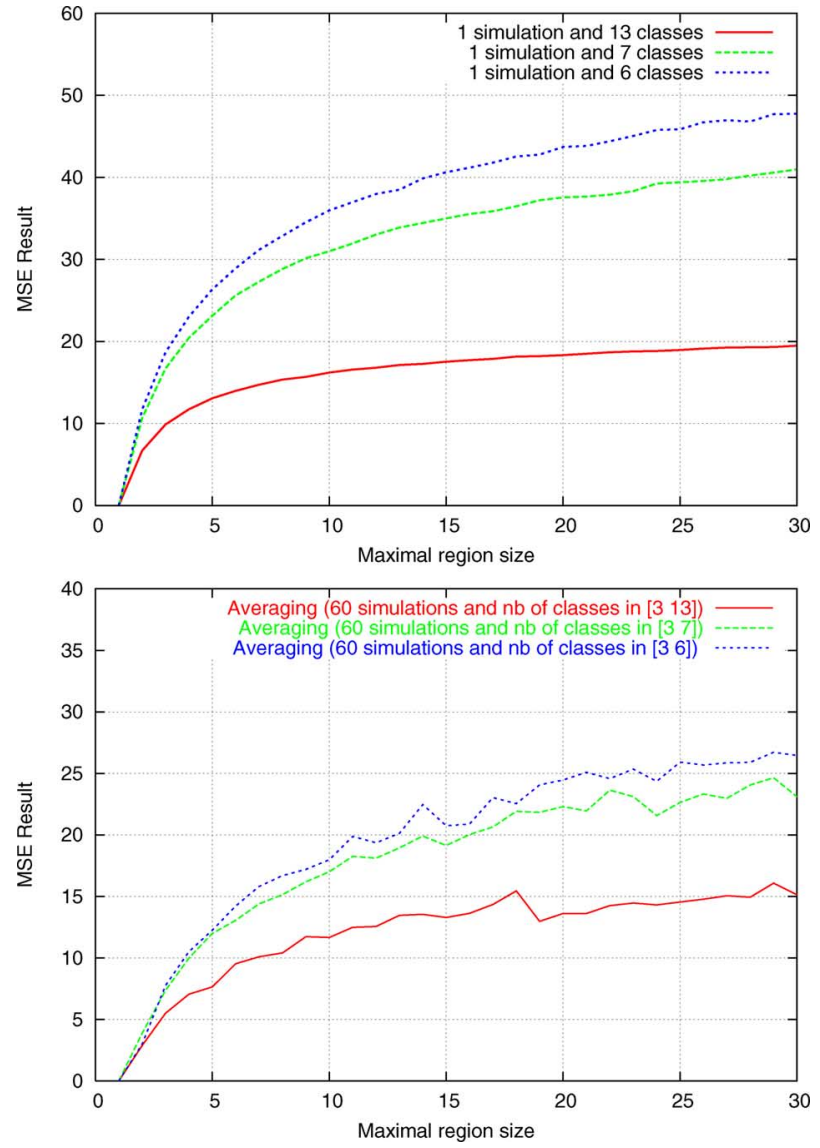

Fig. 5. Evolution of the MSE measure for different maximal region size for respectively (from top to bottom) one and an averaging of 60 simulations.

\section{EXPERIMENTAL RESULTS}

\section{A. Image Model Validity}

First, we can check the validity of our image model expressed in (2). To this end, we compute the MSE error between the unnoisy $512 \times 512$ Lena image and its approximation obtained by our Markovian segmentation procedure under our image model for different maximal region sizes and respectively for a number of classes respectively equals to 13,7 , and 6 (obtained, for example, for a degradation noise respectively equals to $\sigma=10$, 20,30) (see Fig. 5 the MSE as a function of the maximal region size). We have also shown on the second graph, the MSE error between the unnoisy $512 \times 512$ and the averaging of 60 simulations of segmentations as expressed in (5) as a function of the maximal region size and respectively for randomized value of number of classes respectively comprised between [3 - 13], [3 - 7], and [3 - 6] (obtained for example for a degradation noise for which $\sigma=10,20$, or 30). These curves show us both 1) the improvement obtained by the sequential averaging process and 2) a lower bound for the MSE result (for the Lena image) related to each of the considered degradation model. For example, for $\sigma=20$ and , thus, a maximal region size set to $20\left(W_{r}=\sigma\right)$ and number of classes simulated in [3 - 13] (9), a lower bound for the MSE result is approximatively equals to 22 . 

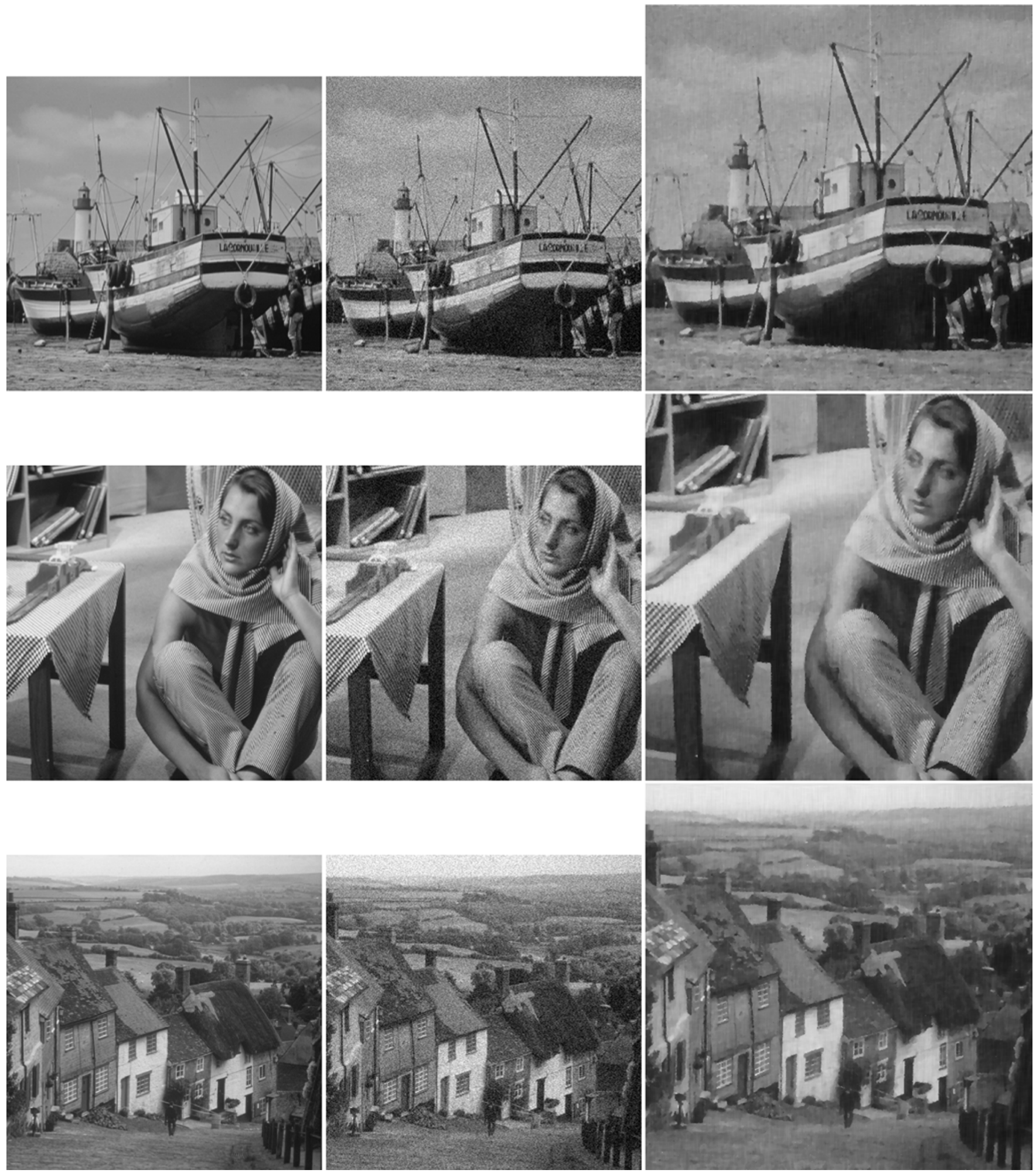

Fig. 6. From left to right, respectively, original $512 \times 512$ image (from top to bottom; Boat, Barbara, and Goldhill), noisy images corrupted with a Gaussian noise of $\sigma=20$ and obtained denoising result with our unsupervised approach (i.e., algorithm USBD) (from top to bottom; $M S E=64.59,153.96,72.63$, see Tables II and IV ).

\section{B. Comparison With State-of-the-Art Methods}

We have considered two variants of our algorithm. First we consider our model for which the internal parameters are given by the empirical estimation given by (7), (9), and $W_{r}=\sigma$ (algorithm called USBD or Unsupervised segmentation-based denoising). Second, we consider our model for which the internal parameters are manually tuned [algorithm called supervised segmentation-based denoising (SSBD)]. For these two algorithms, we consider a sequential averaging process made on 
TABLE I

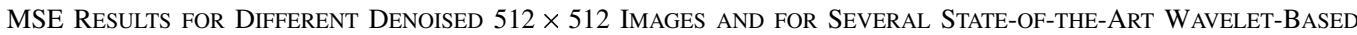
DENOISING METHOdS (ORTHOGONAL TRANSFORMS TO THE LEFT AND REDUNDANT TRANSFORMS TO THE RIGHT)

\begin{tabular}{||c||c|c|c|c|c|c|c||c|c|c|c|c|c|c|c|c|c||}
\hline \hline & $\begin{array}{c}{[1]} \\
\text { in }[2]\end{array}$ & {$[2]$} & {$[13]$} & {$[14]$} & {$[8]$} & {$[9]$} & {$[10]$} & {$[3]$} & {$[6]$} & {$[7]$} & {$[11]$} & {$[12]$} & {$[5]$} & {$[4]$} & {$[38]$} \\
\hline $\begin{array}{l}\text { LENA } \\
\sigma / \text { MSE }\end{array}$
\end{tabular}

TABLE II

MSE RESULTS FOR DIFFERENT DENOISED $512 \times 512$ IMAGES AND FOR SEVERAl RECENT Non WAVELET-BASEd DENOISING Methods.

\begin{tabular}{||c||c|c|c||c|c||}
\hline \hline & $\begin{array}{c}{[39]} \\
\text { (in [23]) }\end{array}$ & {$[23]$} & {$[25]$} & USBD & SSBD \\
\hline $\begin{array}{c}\text { LENA } \\
\sigma / \text { MSE }\end{array}$ & \multicolumn{5}{|c}{} \\
\hline $10 / 100$ & 57.60 & 78.68 & - & 26.44 & 26.23 \\
\hline $15 / 225$ & - & - & - & 44.28 & 40.33 \\
\hline $20 / 400$ & 95.65 & 91.78 & 68.00 & 53.86 & - \\
\hline $25 / 625$ & - & - & - & 65.88 & - \\
\hline $30 / 900$ & 124.55 & 127.9 & - & 77.06 & - \\
\hline BOAT & & & & \\
$\sigma /$ MSE & & & & \\
\hline $10 / 100$ & 58.06 & 129.7 & - & 30.53 & 30.15 \\
\hline $15 / 225$ & - & - & - & 52.00 & 47.37 \\
\hline $20 / 400$ & 145.4 & 150.8 & - & 64.59 & 63.50 \\
\hline $25 / 625$ & - & - & - & 81.89 & 79.52 \\
\hline $30 / 900$ & 228.3 & 186.6 & - & 98.45 & 96.51 \\
\hline \hline
\end{tabular}

$N_{s}=60$ simulations. The overall unsupervised segmentationbased denoising procedure is outlined in pseudo-code in Algorithm I.

We now present a set of experimental results and comparisons illustrating the performance of the proposed supervised or unsupervised approach. For the experiments, we have replicated the degradation models used in the evaluation of state-of-the-art methods described in [1]-[14] and [38] for the wavelet-based methods and [23], [39], [25] for the nonwavelet-based methods. In these experiments, original images are Lena and Boat (of size $512 \times 512$ ) and the variance of the Gaussian noise vary from 100 to 900 . We summarized the MSE results in Tables I and II. Table IV summarized the MSE results for increasing noise degradation model and for other commonly used test $512 \times 512$ images. MSE results for different noise variances obtained on a complex image like Baboon can be compared to the results obtained with wavelet-based denoising methods in [40] and show than our method performs competitively. Fig. 6 shows visually some denoising restorations results on the Boat, Barbara, and Goldhill images after corruption with a Gaussian noise of $\sigma=20$.
TABLE III

TIME IN SECONDS FOR THE PROPOSED Algorithm ON THE DENOISING OF $512 \times 512$ LENA IMAGE $\left(\sigma \in\{10,20,30\}\right.$ AND FOR $\left.N_{s}=60\right)$

\begin{tabular}{||l|l||}
\hline \hline Noise & Time $(\mathrm{sec})$ \\
\hline \hline$\sigma=10$ & $287 \mathrm{sec}$. \\
$\sigma=20$ & $180 \mathrm{sec}$. \\
$\sigma=30$ & $168 \mathrm{sec}$. \\
\hline \hline
\end{tabular}

TABLE IV

MSE RESULTS FOR DIFFERENT DENOISED $512 \times 512$ IMAGES AND FOR OUR UNSUPERVISED AND SUPERVISED (IN BOLD) VERSION OF OUR ALGORITHM (PARAMETER VECTOR [ $\beta$, MAXIMAL Number of Classes and REgion SIZE ] INDICATED BELOW)

\begin{tabular}{|c|c|c|c|}
\hline & \multicolumn{3}{|c|}{$\sigma$} \\
\hline Image & 10 & 20 & 30 \\
\hline \multirow[t]{3}{*}{ ZELDA } & 21.58 & 40.58 & 55.58 \\
\hline & 19.20 & 36.23 & 54.60 \\
\hline & {$[1.20-11-9]$} & {$[1.80-8-18]$} & {$[2.00-7-30]$} \\
\hline \multirow[t]{3}{*}{ BARBARA } & 50.53 & 153.9 & 247.7 \\
\hline & 48.59 & 128.7 & 209.7 \\
\hline & {$[0.68-17-11]$} & {$[0.93-11-21]$} & {$[1.45-11-26]$} \\
\hline \multirow[t]{3}{*}{ PEPPERS } & 26.54 & 51.49 & 75.61 \\
\hline & - & 50.91 & - \\
\hline & & [1.40-10-19] & \\
\hline \multirow[t]{3}{*}{ GOLDHILL } & 36.82 & 72.63 & 105.9 \\
\hline & - & 71.15 & 101.9 \\
\hline & & [1.37-10-11] & [1.60-9-18] \\
\hline \multirow{3}{*}{ BRIDGE } & 69.80 & 165.8 & 250.1 \\
\hline & 58.78 & 139.3 & 210.4 \\
\hline & [0.89-20-4] & [1.38-15-8] & [1.87-14-12] \\
\hline \multirow[t]{3}{*}{ BABOON } & 82.85 & 236.7 & 426.0 \\
\hline & 76.7 & 209.8 & 321.8 \\
\hline & {$[0.38-16-5]$} & {$[0.76-11-16]$} & {$[1.0-8-14]$} \\
\hline \multirow[t]{3}{*}{ F16 } & 26.73 & 61.76 & 96.09 \\
\hline & 26.31 & 60.89 & 91.86 \\
\hline & [0.93-12-9] & [1.81-17-13] & {$[2.00-13-26]$} \\
\hline \multirow[t]{3}{*}{ ELAINE } & 35.87 & 59.77 & 78.24 \\
\hline & - & 58.64 & 75.96 \\
\hline & & [1.91-15-19] & [2.60-11-33] \\
\hline
\end{tabular}

In order to observe the qualitative differences between the state-of-the-art wavelet-based method and our procedure, we have represented in Fig. 7 a detail of the denoised Lena image 

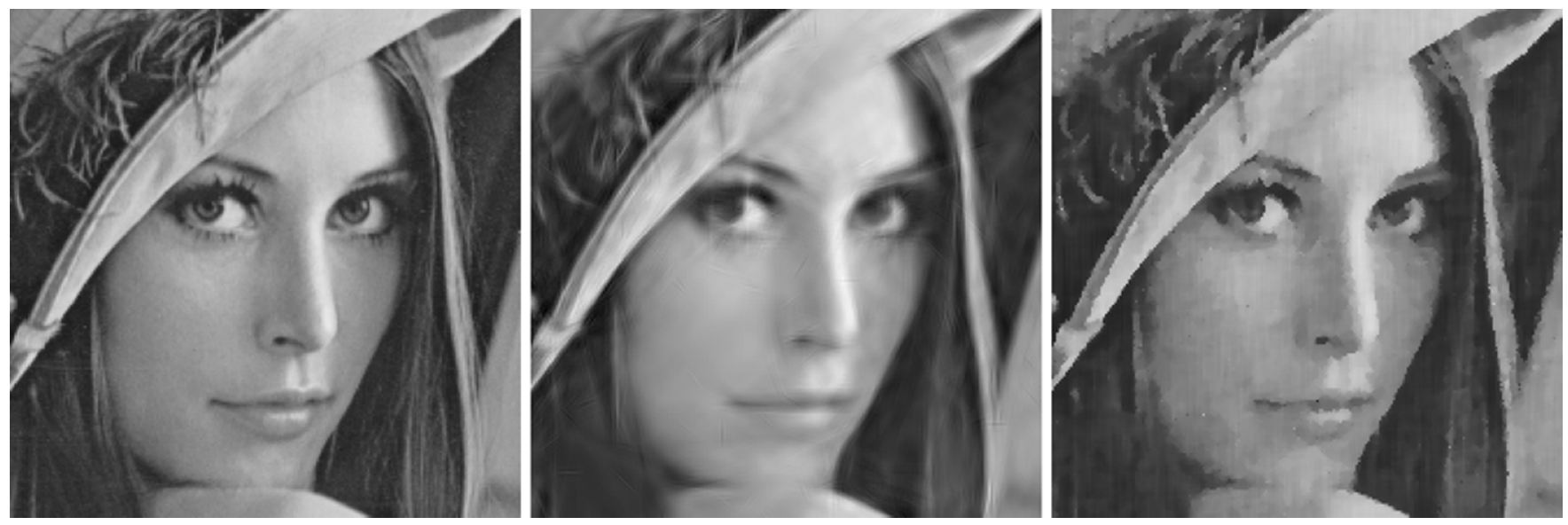

Fig. 7. From top to bottom, respectively, detail of the original $512 \times 512$ Lena image. Denoising result $(\sigma=20)$ obtained with a curvelet-based procedure [7] (example taken from the website of the author at http://www-stat.stanford.edu/ jstarck, MSE $=41.50$ ) and our unsupervised segmentation-based approach MSE $=53.86$.

( $\sigma=20)$ with our method compared to the curvelet-based denoising procedure proposed in [7] (example taken from the website of the author at http://www-stat.stanford.edu/ jstarck) and our denoising approach (see also http: www.iro.umontreal.ca / mignotte/IP-Denoising/ for additional results).

\section{Performance of Our Denoising Process With Other Segmenters}

We have also experimented this segmentation-based denoising approach with a more simple segmentation strategy. To this end, we have only considered a K-means clustering approach (a $K$-class segmenter) for which a stochastic version, required to allow our averaging step, is easily achieved by simply randomly chosen (with different input seeds) the $K$ initial clusters. In this case, the algorithm of our segmentation-based denoising method is the same as the one outlined in pseudo-code in Algorithm 1 but without considering the substeps 2(b)-(d). Two versions of this K-means based segmenter have been tested.

The first one is the classical K-means clustering procedure using grey level intensity as discriminant feature for each pixel. The second one is the previous K-means segmenter followed by a median filter (iterated two times) on the resulting label field proposed by the $\mathrm{K}$-means clustering procedure (i.e., using a majority vote on a squared $3 \times 3$ neighborhood of the segmentation map). This last version allows to easily add a somewhat spatial regularization term that could be interesting in the case of the segmentation of a noisy image which has to be exploited (as in our application) as input of a denoising process. These two versions also exploit our empirical estimation method for $\hat{K}$ and $\hat{W}_{r}$ and use 60 averagings.

We can note that the quality of segmentation is important in the performance of the proposed denoising procedure. The two versions of the $\mathrm{K}$-means denoiser allow herein to reduce the noise, excepted in the case of the Baboon and Barbara images with the second version of the K-means and for $\sigma=10$ for which the resulting MSE is above the initial noise variance (we recall that the resulting MSE gives in fact the variance of the remaining noise on the filtered estimate). The K-means including
TABLE V

MSE RESULTS FOR DIFFERENT DENOISED $512 \times 512$ IMAGES AND FOR OUR DENOISING ALGORITHM (60 AvERAGES) USING From toP TO BOTTOM a K-Means Segmenter and a K-Means Segmenter Followed by A $3 \times 3$ MEDiAn Filter (ITERATED TWO TIMES)

\begin{tabular}{||c||c|c|c||}
\hline \hline \multicolumn{1}{||c||}{} & \multicolumn{3}{c||}{$\sigma$} \\
\hline Image & 10 & 20 & 30 \\
\hline \hline LENA & 56.3 & 234.1 & 590.8 \\
& 32.6 & 59.1 & 93.5 \\
\hline BOAT & 61.9 & 254.6 & 614.1 \\
& 39.7 & 72.7 & 117.1 \\
\hline ZELDA & 55.7 & 246.7 & 628.9 \\
& 20.0 & 42.2 & 73.4 \\
\hline BARBARA & 65.2 & 250.3 & 610.6 \\
& 176.1 & 229.3 & 274.3 \\
\hline PEPPERS & 49.8 & 223.2 & 565.1 \\
& 31.8 & 56.4 & 89.6 \\
\hline BABOON & 79.8 & 308.1 & 668.7 \\
& 309.9 & 401.8 & 473.1 \\
\hline \hline
\end{tabular}

a postprocessing median filtering allows to improve the MSE results compared to a simple K-means but remains less interesting than an unsupervised Markovian segmentation strategy (see Tables IV and V).

This also means that our proposed MRF-segmentation-based denoising model is perfectible and could be improved by using more accurate modeling that the simple Potts-MRF model using Gaussian likelihood distributions as proposed in this paper.

\section{Discussion}

We can note that the proposed method performs very competitively among the nonwavelet-based denoising methods and competitively among the wavelet-based denoising state-of-the-art methods. The unsupervised algorithm gives MSE results relatively close to its supervised version ("-" means that no significant lower MSE result was found with the supervised version of our denoising algorithm) and, thus, shows that the empirical estimators are quite good and reliable excepted for some images with micro textured regions such as Baboon, Bridge, or Barbara for which denoising results in 


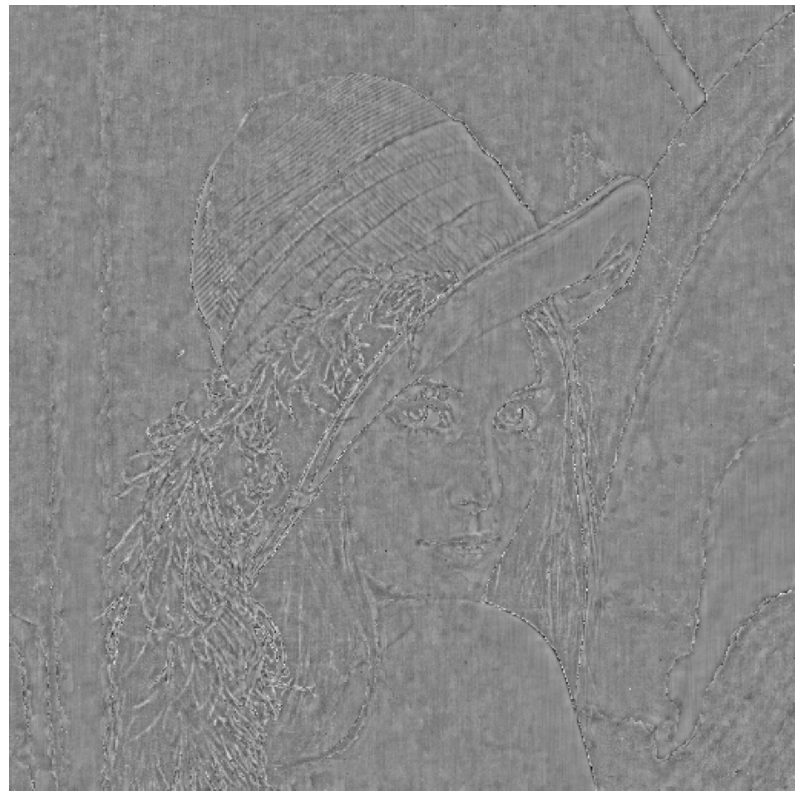

Fig. 8. Method noise MSE $=53.86$ for the Lena image (initially corrupted with AWGN of $\sigma=20$ ) obtained by our unsupervised segmentation-based approach (i.e., algorithm SSBD).

term of MSE is better for values of $\beta$ and $W_{r}$ lower than the empirical estimates.

In our case, a Markovian segmentation model is preferentially used in our segmentation-based denoising procedure for several reasons. First, this statistical segmentation model is especially well suited to take into account the additive independent Gaussian noise that is generally considered in image denoising (and can also be easily adapted to different kinds of noise degradation models such as additive white Weibull or Rayleigh random noise [35]). Second, this model allows to model the local characteristics of the image content thanks to a priori specification of spatial dependencies between neighboring sites (this a priori acts as a regularization term which makes this segmentation robust against noise). Finally, statistical tools (e.g., ICE procedure, adaptive simulated annealing, or MCMC Gibbs sampler) allows us to automatically estimate (in a statistical criterion sense) the parameters of any MRF segmentation models.

We have also computed the residual noise after a denoising obtained by our method and displayed the result in Fig. 8. The property of this residual noise (also called the method noise) tells us which geometrical features or details are preserved by the denoising process and which are eliminated [25] and, thus, is a important criterion in order to qualitatively judge the performance of a denoising algorithm.

Table III shows the time in seconds that our algorithm took for a total of 60 simulations and for each considered degradation model (system used: AMD Athlon 64 Processor 3500+, 2.2-GHz, 4374 bogomips and running on Linux). Let us note that our denoising system can be easily implemented in parallel and that three times less of simulations (for three times less of computational cost) would lead to a quasi convergence (and would lead to a similar MSE result) for our method (see Fig. 4).
MRF Segmentation-Based Denoising Algorithm

$\mid \begin{array}{ll}\sigma^{2} & \text { Variance of the degradation noise } \\ N_{\mathrm{S}} & \text { Number of segmentations/partitions } \\ K & \text { Number of classes of the segmentation } \\ \beta & \text { Regularization parameter of the posterior } \\ W_{r} & \text { Maximal size of each region }\end{array}$

\section{Initialization}

$$
\beta \leftarrow \max \{0.25, \sigma / 20\} \quad W_{r} \leftarrow \sigma
$$

2. Unsupervised Oversegmentations/Partitions for $k=1$ to $N_{s}$ do

$$
\begin{gathered}
K \leftarrow \text { Random Integer in }[3, \max \{6,256 / 2 \sigma\}] \\
\text { according to a uniform law }
\end{gathered}
$$

(a) K-means clustering of each pixel of $y$ into $K$ clusters, initially randomly chosen $(\operatorname{SEED}=k)$

(b) $\Phi_{y} \leftarrow$ Maximum Likelihood (ML) fitting of a Gaussian law $\mathcal{G}\left(\mu, \sigma^{2}\right)$ for each cluster $\left(\Phi_{y}=\left[\mu_{1}, \sigma_{1}^{2}, \ldots, \mu_{K}, \sigma_{K}^{2}\right]\right)$

(c) $z_{\mathrm{ML}} \leftarrow$ ML segmentation of $y$ into $K$ classes based on $\Phi_{y}$

(d) $z \leftarrow$ ICE algorithm on $y$ (initialized with $z_{\mathrm{ML}}$, $\Phi_{y}$ and with $\operatorname{SEED}=k$ )

(e) $x_{(k)} \leftarrow$ Partition of $z$ into $N_{\mathrm{R}}$ distinct regions with randomly chosen patterns of subdivision (of less than $W_{r}$ pixel size) in which the set of pixel values belonging to the $j^{\text {th }}$ region $\mathcal{R}_{j}$ is replaced by its mean value of grey level $\mu_{j}$ i.e.,

$$
x_{i(k)}=\sum_{j=1}^{N_{\mathrm{R}}} \mu_{j} I_{\left\{i \in \mathcal{R}_{j}\right\}}, \quad \text { for } \quad i=1, \cdots, N
$$

3. Segmentation-Based Denoising

$$
\hat{x} \leftarrow \frac{1}{N_{\mathrm{s}}} \sum_{k=1}^{N_{\mathrm{S}}} x_{(k)}
$$

Algorithm 1. MRF Segmentation-based denoising algorithm.

\section{CONCLUSION}

In this paper, we have presented a new denoising method which is based on a sequential averaging of piecewise uniform simulated image partitions. These simulations are obtained by a Gibbs sampler of possible oversegmentations for which internal parameters are automatically and empirically estimated. The proposed segmentation-based denoising technique efficiently adapts to local characteristics of the data and allows to reduce the noise while preserving contours and texture of the original image. The proposed method challenges better among existing nonwavelet-based denoising techniques and performs competitively among the recently reported state-of-the-art denoising systems using wavelet decompositions. This method also proposes a new spatial domain promising avenue for the denoising problem for which it is interesting to speculate how the performance of such segmentation-based denoising system might be improved. In that prospect, the denoising model could be improved by using more accurate modeling for the segmentation process since the Gaussian likelihood model used in our segmentation process is the simplest one. In addition a more accurate prior model could be also take into account. To this end, Bayesian theory makes it possible to apply (or to add to the classical Potts local prior model) 
Subdivisions Into Randomized Patterns

$W_{r} \quad$ Maximal size of each subdivision pattern cpt Counter

for each set of connected pixel belonging to the same class $e_{k}$ of $z_{(i)}$ and that does not belong to any previous patterns of subdivision do

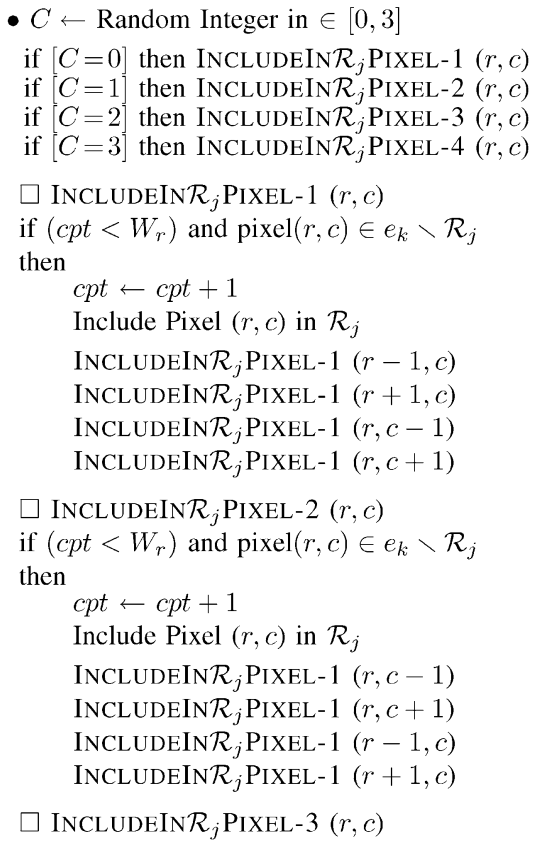

Algorithm 2. Partition of a set of connected pixels belonging to the same class of $z_{(i)}$ into distinct regions with randomly chosen patterns of subdivision.

global prior spatial constraints, such as the size, the number of regions or the smoothness of the region boundaries, the shape of these regions, etc., which may be also expressed by a probabilistic models in order to more accurately constraint the segmentation problem [41], [42]. A better and truly statistical estimation procedure for the regularization parameters of this method could be also investigated. It would also be interesting to consider a more realistic image model; and to consider that an image is certainly more rightly approximated by a sum of $N_{R}$ piecewise polynomial (and not constant) functions. Let us also note that the proposed model can be easily adapted, with simulation of spatio-temporal oversegmentations, for the denoising of video image sequences. Let us finally add that this sequential averaging-based process can easily be combined with other complementary spatial domain denoising procedures such as the fractal-based [23] algorithm or the non local means procedure [25] recently proposed in order to obtain further restoration improvements.

\section{ACKNOWLEDGMENT}

The author would like to thank the reviewers and Dr. F. Destrempes for their many valuable comments and suggestions that helped to improve both the technical content and the presentation quality of this paper.

\section{REFERENCES}

[1] D. L. Donoho and I. M. Johnstone, "Ideal spatial adaptation by wavelet shrinkage," Biometrika, vol. 81, pp. 425-455, 1994

[2] M. K. Mihcak, I. Kozintsev, K. Ramchandran, and P. Moulin, "Lowcomplexity image denoising based on statistical modeling of wavelet coefficients," IEEE Signal Process. Lett., vol. 6, no. 12, pp. 300-303, Dec. 1999.

[3] S. G. Chang, B. Yu, and M. Vetterli, "Spatially adaptive wavelet thresholding with context modeling for image denoising," IEEE Trans. Image Process., vol. 9, no. 9, pp. 1522-1531, Sep. 2000.

[4] L. Sendur and I. W. Selesnick, "Bivariate shrinkage with local variance estimation," IEEE Signal Process. Lett., vol. 9, no. 12, pp. 438-441, Dec. 2002.

[5] G. Fan and X.-G. Xia, "Image denoising using a local contextual hidden Markov model in the wavelet domain," IEEE Signal Process. Lett., vol. 8, no. 5, pp. 125-128, May 2001.

[6] L. Sendur and I. W. Selesnick, "Bivariate shrinkage for wavelet-based denoising," IEEE Trans. Signal Process., vol. 50, no. 11, pp. 2744-2756, Nov. 2002.

[7] J.-L. Starck, E. J. Candes, and D. L. Donoho, "The curvelet transform for image denoising," IEEE Trans. Image Process., vol. 11, no. 6, pp. 670-684, Jun. 2002.

[8] J. Liu and P. Moulin, "Complexity-regularized image denoising," IEEE Trans. Image Process., vol. 10, no. 6, pp. 841-851, Jun. 2001.

[9] H. Choi and R. G. Baraniuk, "Multiple wavelet basis image denoising using Besov ball projections," IEEE Signal Process. Lett., vol. 11, no. 9, pp. 717-720, Sep. 2004.

[10] M. Kazubek, "Wavelet domain image denoising by thresholding and Wiener filtering," IEEE Signal Process. Lett., vol. 10, no. 11, pp. 324-326, Nov. 2003.

[11] J. Portilla, V. Strela, M. J. Wainwright, and E. P. Simoncelli, "Image denoising using scale mixtures of Gaussians in the wavelet domain," IEEE Trans. Image Process., vol. 12, no. 11, pp. 1338-1351, Nov. 2003.

[12] A. Achim and E. E. Kuruoglu, "Image denoising using bivariate $\alpha$-stable distributions in the complex wavelet domain," IEEE Signal Process. Lett., vol. 12, no. 1, pp. 17-20, Jan. 2005.

[13] I. K. Eom and Y. S. Kim, "Wavelet-based denoising with nearly arbitrarily shaped windows," IEEE Signal Process. Lett., vol. 11, no. 12, pp. 937-940, Dec. 2004.

[14] M. Singh, P. Ishwar, K. Ratakonda, and N. Ahuja, "Segmentation based denoising using multiple compaction domains," in Proc. IEEE Int. Conf. Image Processing, Oct. 1999, vol. 1, pp. 372-375.

[15] F. Abramovich, T. Sapatinas, and B. Silverman, "Wavelet thresholding via a Bayesian approach," J. Roy. Statist. Soc. B, vol. 60, pt. 4, pp. 725-749, 1998.

[16] H. A. Chipman, E. D. Kolaczyk, and R. E. McCulloch, "Adaptive Bayesian wavelet shrinkage," J. Amer. Statist. Assoc., vol. 92, pp. 1413-1421, 1997.

[17] B. Vidakovic, "Nonlinear wavelet shrinkage with Bayes rules and Bayes factors," J. Amer. Statist. Assoc., vol. 93, pp. 173-179, 1998.

[18] M. Clyde, G. Parmigiani, and B. Vidakovic, "Multiple shrinkage and subset selection in wavelets," Biometrika, vol. 85, no. 2, pp. 391-401, 1998.

[19] B. Vidakovic, "Wavelet-based nonparametric Bayes methods," Lecture Notes Statist., no. 133, pp. 133-155, 1998.

[20] A. Bijaoui, "Wavelets, Gaussian mixtures and Wiener filtering," Signal Process., vol. 82, no. 4, pp. 709-712, 2002.

[21] H. Choi, J. Romberg, R. Baraniuk, and N. Kingsbury, "Hidden Markov tree modeling of complex wavelet transforms," presented at the IEEE Int. Conf. Acoustics, Speech, Signal Processing, Jun. 2000.

[22] T. C. M. Lee, "A minimum description length based image segmentation procedure, and its comparison with a cross-validation based segmentation procedure," J. Amer. Statist. Assoc., vol. 95, pp. 259-270, 2000 .

[23] M. Ghazel, G. H. Freeman, and E. R. Vrscay, "Fractal image denoising," IEEE Trans. Image Process., vol. 12, no. 12, pp. 1560-1578, Dec. 2003.

[24] K. U. Barthel, H. L. Cycon, and D. Marpe, "Image denoising using fractal and wavelet-based methods," presented at the SPIE's Int. Symp. Photonics Technologies for Robotics, Automation, and Manufacturing Wavelet Applications in Industrial Processing, Oct. 2003.

[25] A. Buades, B. Coll, and J.-M. Morel, "On image denoising methods," SIAM Multiscale Modeling and Simulation, 2005, to be published.

[26] A. Efros and T. Leung, "Texture synthesis by non-parametric sampling," in Proc. Int. Conf. Coputer Vision, 1999, pp. 1033-1038. 
[27] D. Mumford and J. Shah, "Optimal approximations by picewise smooth functions and associated variational problems," Сотmun. Pure Appl. Math., vol. 42, no. 5, pp. 577-685, 1989.

[28] S. Voloshynovskiy, O. Koval, and T. Pun, "Wavelet-based image denoising using non-stationary stochastic geometrical image priors," presented at the SPIE Image and Video Communications and Processing V, Santa Clara, CA, Jan. 2003.

[29] T. Seeman and P. Tischer, "Structure preserving noise filtering of images," in Proc. Int. Conf. Pattern Recognition, Aug. 1998, vol. 2, pp. $1610-1612$.

[30] M. W. Hansen and W. E. Higgins, "Image enhancement using watershed-based maximum homogeneity filtering," in Proc. IEEE Int. Conf. Image Processing, 1995, vol. II, pp. 482-485.

[31] M. Feigin and N. Sochen, "Segmentation and denoising via an adaptive threshold Mumford-Shah-like functional," in Proc. IEEE Int. Conf. Pattern Recognition, Aug. 2004, vol. 2, pp. 98-101.

[32] M. Mignotte, "A segmentation-based regularization term for image deconvolution," IEEE Trans. Image Process., vol. 15, no. 7, pp. 1973-1984, Jul. 2006.

[33] $\_$, "An adaptive segmentation-based regularization term for image restoration," in Proc. IEEE Int. Conf. Image Processing, Sep. 2005, vol. 1, pp. 901-904.

[34] R. Gonzales and R. Woods, Digital Image Processing. Reading, MA: Addison-Wesley, 1992.

[35] M. Mignotte, C. Collet, P. Pérez, and P. Bouthemy, "Sonar image segmentation using a hierarchical MRF model," IEEE Trans. Image Process., vol. 9, no. 7, pp. 1216-1231, Jul. 2000.

[36] B. Braathen, P. Masson, and W. Pieczynski, "Global and local methods of unsupervised Bayesian segmentation of images," Graph. Vis., vol. 2, no. 1, pp. 39-52, 1993.

[37] S. Banks, Signal Processing, Image Processing and Pattern Recognition. Englewood Cliffs, NJ: Prentice-Hall, 1990.

[38] A. A. Bharath and J. Ng, "A steerable complex wavelet construction and its application to image denoising," IEEE Trans. Image Process., vol. 14, no. 7, pp. 948-959, Jul. 2005.
[39] J. S. Lee, "Digital image enhancement and noise filtering by use of local statistics," IEEE Trans. Pattern Anal. Mach. Intell., vol. PAMI-2, no. 2, pp. 165-168, Feb. 1980.

[40] S. G. Chang, B. Yu, and M. Vetterli, "Adaptive wavelet thresholding for image denoising and compression," IEEE Trans. Image Process., vol. 9, no. 9, pp. 1532-1546, Sep., 2000.

[41] Z. Tu and S.-C. Zhu, "Image segmentation by data-driven Markov chain Monte-Carlo," IEEE Trans. Pattern Anal. Mach. Intell., vol. 24, no. 5, pp. 657-673, May 2002.

[42] A. Lee, D. B. Mumford, and J. Huang, "Occusion models for natural images," Int. J. Comput. Vis., vol. 41, pp. 35-59, 2001.

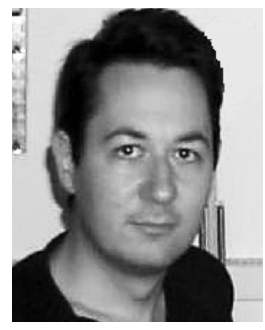

Max Mignotte received the DEA (postgraduate degree) in digital signal, image, and speech processing from the INPG University, Grenoble, France, in 1993, and the Ph.D. degree in electronics and computer engineering from the University of Bretagne Occidentale (UBO) and the digital signal laboratory (GTS) of the French Naval academy, France, in 1998.

He was an INRIA Postdoctoral Fellow at University of Montréal (DIRO), Montréal, QC, Canada, from 1998 to 1999 . He is currently with the Computer Vision and Geometric Modeling Lab, DIRO, as an Assistant Professor (Professeur adjoint). He is also a member of the Laboratoire de recherche en imagerie et orthopedie (LIO), Centre de recherche du CHUM, Hopital Notre-Dame, and a Researcher at CHUM. His current research interests include statistical methods and Bayesian inference for image segmentation (with hierarchical Markovian, statistical templates, or active contour models), hierarchical models for high-dimensional inverse problems from early vision, parameters estimation, tracking, classification, shape recognition, deconvolution, 3-D reconstruction, and restoration problems. 